\title{
Constituent Properties of Licorices Derived from Glycyrrhiza uralensis, G. glabra, or G. inflata Identified by Genetic Information
}

\author{
Kenji Kondo, ${ }^{*, a}$ Mao Shiba, ${ }^{a}$ Rie Nakamura, ${ }^{a}$ Takashi Morota, ${ }^{a}$ and Yukihiro Shoyama ${ }^{b}$ \\ ${ }^{a}$ Botanical Raw Materials Research Dept., Tsumura \& Co.; Ibaraki 300-1192, Japan: and ${ }^{b}$ Graduate School of \\ Pharmaceutical Sciences, Kyushu University; Fukuoka 812-8582, Japan.
}

Received January 6, 2007; accepted March 26, 2007

\begin{abstract}
Constituent properties of licorices derived from Glycyrrhiza uralensis, G. glabra, and G. inflata are revealed by comparing 117 of licorice identified using four genetic markers; internal tracscribed spacer (ITS) on nuclear ribosomal DNA, rbcL gene, matK gene, and trnH-trnK1 intergenic region on chloroplast DNA. Regarding six main constituents of licorice; glycyrrhizin, liquiritin, liquiritin apioside, isoliquiritin, isoliquiritin apioside, and liquiritigenin, the constituent property of G. glabra resembles to that of $G$. inflata. On the other hand, the constituent property of $G$. uralensis is not similar to that of $G$. glabra or $G$. inflata and is characterized by a wide content variation of the six constituents compared to those of G. glabra and/or G. inflata. The mean contents of liquiritin, isoliquiritin, or liquilitigenin in G. uralensis are significantly higher than those of G. glabra or G. inflata. Therefore, the licorice species should be selected depending on these constituent properties for the traditional Chinese medicines or the Japanese Kampo medicines. Additionally, glycycoumarin, glabridin, and licochalcone A were reconfirmed as the species-specific typical constituents of G. uralensis, G. glabra, and G. inflata respectively. Therefore, it is resulted that the determination of the three species-specific constituents may be useful for the species identification of licorice. However, since $6 \%$ of licorice examined and hybrids were exceptions to the rule, their genetic information is necessary for the accurate species identification of licorice.
\end{abstract} stituent

Key words licorice; Glycyrrhiza uralensis; Glycyrrhiza glabra; Glycyrrhiza inflata; constituent property; species-specific con-

Licorice is one of the most important herbal medicines for the traditional Chinese medicine and the Japanese Kampo medicine. In Chinese Pharmacopoeia, three species of Glycyrrhiza uralensis, G. glabra and G. inflate are listed as licorice. ${ }^{1)}$ While in Japanese Pharmacopoeia, two species of G. uralensis and G. glabra are prescribed. ${ }^{2)}$

Constituent properties of licorices derived from the three species have been reported about eleven kinds of constituent contents, ${ }^{3,4)}$ HPLC patterns, ${ }^{5,6)}$ and species-specific constituents of glycycoumarin, glabridin, or licochalcone A. ${ }^{7-9)}$ However, it was difficult to clarify differences of constituent properties among licorices derived from the three species because the accurate species identification of licorice was difficult from their morphological features of medicinal parts, and existences of hybrids among Glycyrrhiza species had been reported, too. ${ }^{10-12)}$ Additionally, a number of licorices identified exactly has been offered insufficiently for those constituent analyses. Recently, a method of species identification of licorice using four kinds of genetic markers; internal tracscribed spacer (ITS) on nuclear ribosomal DNA, $r b c L$ gene, matK gene, and trnH-trnK1 intergenic region on chloroplast DNA, has been reported. ${ }^{13)}$ This method would be able to detect the hybrid between $G$. uralensis and, $G$. glabra or G. inflata from the ITS sequence of nuclear ribosomal DNA.

Therefore, to clarify the differences of constituent properties among the licorices derived from the three species, nucleotide sequences of the four kinds of DNA region of 117 licorices were researched and their species were identified at first. Secondly, contents of six kinds of main constituents of licorice; glycyrrhizin, liquiritin, liquiritin apioside, isoliquiritin, isoliquiritin apioside, and liquiritigenin, ${ }^{3)}$ and three kinds of the species-specific constituents; glycycoumarin, glabridin, and licochalcone $\mathrm{A},{ }^{7-9)}$ were measured in the 117 licorices. Consequently, the constituent properties by the licorice derived from the three species will be suggested by comparing these nine kinds of constituent contents of licorices classified by the three species based on the genetic information.

\section{MATERIALS AND METHODS}

Materials Vouchers and localities of 117 licorices are listed in Table 1. The vouchers are deposited in the herbarium of Tsumura \& Co. (THS) in Japan.

DNA Sequencing Total DNA was extracted by using DNAeasy ${ }^{\mathbb{B}}$ Plant Mini Kit (QIAGEN) from the medicinal part of licorice. The complete sequence of the ITS and the partial sequences of $r b c L(168 \mathrm{bp}), \operatorname{matK}(143 \mathrm{bp})$, and trnH-trnK1 (178 bp) including the genetic polymorphism were amplified by PCR with the following condition. Amplifying primers for ITS were ITS5: GGA AGT AAA AGT CGT AAC AAG G and ITS4: TCC TCC GCT TAT TGA TAT GC. ${ }^{14)}$ The amplifying primers for $r b c L$ were $\mathrm{r} 662 \mathrm{f}$ : GTG CCG AAG CAA TTT ATA AAG C and r829r: TTG CAG TGA AAC CTC CAG TT, for $m a t K$ were m1242f: CTT CGA CAC TGG GTG AAA GAT G and m1384r: AGG AAC AAG AAT AAT CTT GG, and for trnH-trnKl were trnH-forward: ACG GGA ATT GAA CCC GCG $\mathrm{CA}^{15)}$ and Gly-trnHR1: CAT ATG ACT TCA CAA TGT AAA ATC. The PCR reaction mixture was as follows: $10 \times$ Gene Taq Buffer (Nippon Gene) $5 \mu \mathrm{l}$, dNTP mix (Nippon Gene) $4 \mu \mathrm{l}$, forward Primer $(10 \mathrm{pmol} / \mathrm{ml}) 2.5 \mu \mathrm{l}$, reverse Primer $(10$ $\mathrm{pmol} / \mathrm{ml}$ ) $2.5 \mu \mathrm{l}$, Gene Taq (Nippon Gene), DMSO $5 \mu \mathrm{l}$, D.D.W. $25.75 \mu$ l, template DNA $5 \mu \mathrm{l}(5 \mathrm{ng})$. For the PCR cycle, a modified protocol of the Step Down PCR was applied. ${ }^{16)}$

Electrophoreses were performed for the amplified DNA 
fragments through $1.2 \%$ TAE agarose gel. The amplified DNA fragments were cut out from the gel and purified using the GFX ${ }^{\mathrm{TM}}$ PCR DNA and Gel Band Purification Kit (Amersham biotech). The purified PCR products were sequenced using the BigDye Terminator Cycle Sequencing Kit ver.2.0 and Model 3100 automated sequencer (Applied biosystems) following the manufacturer's instructions. For sequencing the ITS, matK, and trnH-trnK1, their amplifying primers were used as sequencing primers. For sequencing the $r b c L$, r694f: ACT GGT GAA ATC AAA GGG C and r809r: AAG TAG TCA TGC ATT ACG AT were designed as sequencing primers.

Species Identification In the species identification of licorice using the four kinds of genetic markers, ten kinds of the total genotypes $(\operatorname{tg} 1-\operatorname{tg} 9$, and ADD) have been recognized. ${ }^{13)}$ Additionally, they have been suggested in the species identification of licorice that $\operatorname{tg} 1$ is the genotype of Glycyrrhiza species except $G$. uralensis, G. glabra, or $G$. inflata, tg2 is G. glabra or G. inflata, tg3 is G. glabra, tg4 and $\operatorname{tg} 5$ are $G$. inflata, $\operatorname{tg} 6$ - $\operatorname{tg} 9$ are $G$. uralensis, and ADD is hybrid between $G$. uralensis and $G$. glabra, or $G$. inflata.

Chemical Analysis The powders of licorice $(0.05 \mathrm{~g})$ were extracted with $80 \%$ methanol $(25 \mathrm{ml})$ under ultrasonication for $30 \mathrm{~min}$. This operation was repeated and scaled up to $50 \mathrm{ml}$. The $20 \mu \mathrm{l}$ of this extract was submitted to HPLC analysis. HPLC equipped with a LC-10 system (Shimadzu) using a TSK gel ODS-80T $\mathrm{T}_{\mathrm{S}}$ column $(250 \times 4.6 \mathrm{~mm})$ was used. The solvents were $0.05 \mathrm{M} \mathrm{AcONH}_{4}(\mathrm{pH} 3.6)(\mathrm{A})$ and $\mathrm{CH}_{3} \mathrm{CN}(\mathrm{B})$. A linear gradient of $100 \%$ (A) and $0 \%$ (B) changing over $60 \mathrm{~min}$. to $0 \%(\mathrm{~A})$ and $100 \%(\mathrm{~B})$ was used. The flow rate was $1.0 \mathrm{ml} / \mathrm{min}$. The effluent from the column was monitored at from $200 \mathrm{~nm}$ to $400 \mathrm{~nm}$.

Statistical Analysis A principal component analysis for the contents of the six main constituents of licorice; glycyrrhizin, liquiritin, liquiritin apioside, isoliquiritin, isoliquiritin apioside, liquiritigenin, were performed using the Excel statistics 2002 for windows software. ${ }^{15)}$

\section{RESULTS}

Species Identification Using the Four Kinds of Genetic Markers Six kinds of the genotypes $(\operatorname{tg} 3, \operatorname{tg} 5, \operatorname{tg} 7, \operatorname{tg} 8, \operatorname{tg} 9$, and $\mathrm{ADD})^{13)}$ were recognized by the genetic analyses for the 117 licorices (Table 1). Seventeen licorices located in Russia, France, Holland, Germany, Iran, Afghanistan, and Xinjiang in China had the $\operatorname{tg} 3$ genotype and were identified as $G$. glabra. Eight licorices in Xinjiang and Gansu in China had the $\operatorname{tg} 5$ genotype and were identified as $G$. inflata. Eighty seven licorices in Russia and a large north area from Jilin to Xinjiang in China had the $\operatorname{tg} 7(n=14), \operatorname{tg} 8(n=19)$, and $\operatorname{tg} 9$ $(n=54)$ genotypes and were identified as $G$. uralensis. Five licorices in Xinjiang and Gansu in China had the ADD genotype and were identified as hybrids between $G$. uralensis and G. glabra, or G. inflata.

The Six Kinds of Main Constituent Contents by the Three Species Used as Licorice The contents of the six kinds of main constituents; glycyrrhizin, liquiritin, liquiritin apioside, isoliquiritin, isoliquiritin apioside, and liquiritigenin, in the 117 licorices examined were indicated in Table 1. Comparing mean contents of the six main constituents by G. uralensis, G. glabra, or G. inflata identified from their ge- netic information, there was no significant difference among the three species regarding the contents of glycyrrhizin, liquiritin apioside, or isoliquiritin apioside. While, the mean contents of liquiritin, isoliquiritin, and liquilitigenin in $G$. uralensis were significantly higher than those of G. glabra or G. inflata (Fig. 1). However, the mean content of isoliquiritin or liquiritigenin in G. uralensis was only $0.32 \%$ or $0.11 \%$ respectively. Liquiritin in G. uralensis only had more than $1 \%$ higher content than that of G. glabra or G. inflata.

In the result of a principal component analysis for the six main constituent contents, the licorices derived from $G$. glabra and $G$. inflata were located together on the third quadrant of the scatter diagram mainly, while $G$. uralensis were scattered on all of the quadrants (Fig. 2). It means that the content property of the six main constituents of G. glabra resembles to $G$. inflata. On the other hand, the content property of the six main constituents of $G$. uralensis does not resemble to G. glabra or G. inflata, and is characterized by a wider content variation than that of G. glabra or G. inflata.

In $G$. uralensis, the three kinds of genotypes $(\operatorname{tg} 7, \operatorname{tg} 8$, and $\operatorname{tg} 9)$ were recognized as an intra-specific variation. There is no difference of content properties among licorices having the three genotypes on the scatter diagram of the principal component analysis. Additionally, five licorices derived from hybrids with the ADD genotype located at the intermediate area in the distributions of the three species on the scatter diagram (Fig. 2).

Species-Specific Constituents of Licorice Glycycoumarin, glabridin, or licochalcone A had been reported as the species-specific constituents of G. uralensis, G. glabra, or $G$. inflata respectively. ${ }^{7-9)}$ Their constituent contents in the 117 licorices examined were indicated in Table 1. The detection frequencies of the three kinds of the species-specific constituents by the three species and hybrid identified from the genetic information were indicated in Table 2 . In $G$. glabra $(n=17), 88 \%(n=15)$ had glabridin and $12 \%(n=2)$ had no species-specific constituents. In $G$. inflata $(n=8)$, $88 \%(n=7)$ had licochalcone A and 12\% $(n=1)$ had no species-specific constituent. In G. uralensis $(n=87), 95 \%$ $(n=83)$ had glycycoumarin and 5\% $(n=4)$ had no speciesspecific constituent. In hybrids between $G$. uralensis and $G$. glabra, or $G$. inflata $(n=5), 40 \%(n=2)$ had glycycoumarin and $60 \%(n=3)$ had licochalcone A. Therefore, it is reconfirmed that glabridin, licochalcon A, or glycycoumarin functions as a species-specific constituents of G. glabra, G. inflata, or $G$. uralensis respectively because the each constituent was recognized in only G. glabra, G. inflata, or $G$. uralensis identified by the genetic information respectively.

However, in the hybrids identified by their genetic information, two kinds of the species-specific constituents derived from their both parents were not detected from an individual but only one kind of species-specific constituent was detectable (Table 1). Additionally, Hayashi et al. had been reported that glabridin or glycycoumarin were not detected from the licorices in Kazakhstan that would be identified as hybrids between $G$. glabra and $G$. uralensis from their leaf shapes, ${ }^{11)}$ and glycycoumarin was detected from the licorices cultivated in Japan, although they had the AT genotype $(=G$. glabra) or GA genotype (=G. uralensis) of $r b c L$ gene. ${ }^{12)}$ In this regard, Hayashi et al. had suggested that introgressive hybridization between G. uralensis and G. glabra resulted in 
Table 1. Vouchers, Locations, Species Identified by their Genotypes, and Contents of the Six Kinds of Main Constituents of Licorice and the Three Kinds of Species-Specific Constituents for 117 Licorices

\begin{tabular}{|c|c|c|c|c|c|c|c|c|c|c|c|c|}
\hline \multirow{2}{*}{ Voucher } & \multirow{2}{*}{ Locality } & \multirow{2}{*}{$\begin{array}{l}\text { Geno- } \\
\text { type }\end{array}$} & \multirow{2}{*}{$\begin{array}{c}\text { Species } \\
\text { identified } \\
\text { by } \\
\text { genotype }\end{array}$} & \multicolumn{6}{|c|}{$\begin{array}{l}\text { Contents of the six main } \\
\text { constituents of licorice (\%) }\end{array}$} & \multicolumn{3}{|c|}{$\begin{array}{l}\text { Contents of the species- } \\
\text { specific constituens (\%) }\end{array}$} \\
\hline & & & & $\begin{array}{l}\text { Glycyr- } \\
\text { rhizin }\end{array}$ & Liquiritin & $\begin{array}{l}\text { Liquiritin } \\
\text { apioside }\end{array}$ & $\begin{array}{l}\text { Isoliqui- } \\
\text { ritin }\end{array}$ & $\begin{array}{l}\text { Isoliquiritin } \\
\text { apioside }\end{array}$ & $\begin{array}{l}\text { Liquiriti- } \\
\text { genin }\end{array}$ & $\begin{array}{l}\text { Glycycou- } \\
\text { marin }\end{array}$ & Glabridin & $\begin{array}{l}\text { Licochal- } \\
\text { cone A }\end{array}$ \\
\hline THS60130 & Russia & $\operatorname{tg} 3$ & G. glabla & 5.860 & 0.112 & 2.131 & n.d. & 0.838 & n.d. & n.d. & 0.273 & n.d. \\
\hline THS61104 & Russia & $\operatorname{tg} 3$ & G. glabla & 3.787 & 0.097 & 1.217 & n.d. & 0.515 & n.d. & n.d. & 0.136 & n.d. \\
\hline THS61121 & Russia & $\operatorname{tg} 3$ & G. glabla & 7.007 & 0.232 & 1.612 & n.d. & 0.625 & 0.043 & n.d. & 0.045 & n.d. \\
\hline THS66496 & Spain & $\operatorname{tg} 3$ & G. glabla & 2.787 & 0.107 & 2.743 & n.d. & 1.021 & 0.021 & n.d. & 0.247 & n.d. \\
\hline THS66495 & France & $\operatorname{tg} 3$ & G. glabla & 2.666 & 0.078 & 2.674 & n.d. & 0.213 & n.d. & n.d. & 0.102 & n.d. \\
\hline THS66497 & Holland & $\operatorname{tg} 3$ & G. glabla & 4.206 & 0.144 & 1.802 & n.d. & 0.699 & 0.023 & n.d. & 0.239 & n.d. \\
\hline THS66219 & Germany & $\operatorname{tg} 3$ & G. glabla & 1.875 & 0.134 & 0.723 & 0.047 & 0.240 & 0.022 & n.d. & 0.055 & n.d. \\
\hline THS66221 & Germany & $\operatorname{tg} 3$ & G. glabla & 4.842 & 0.106 & 1.612 & n.d. & 0.616 & n.d. & n.d. & 0.026 & n.d. \\
\hline THS54514 & Iran & $\operatorname{tg} 3$ & G. glabla & 2.301 & 0.035 & 0.904 & n.d. & 0.339 & n.d. & n.d. & 0.056 & n.d. \\
\hline THS64261 & Iran & $\operatorname{tg} 3$ & G. glabla & 3.481 & 0.008 & 1.373 & n.d. & 0.455 & 0.010 & n.d. & n.d. & n.d. \\
\hline THS60101 & Afghanistan & $\operatorname{tg} 3$ & G. glabla & 5.384 & 0.096 & 1.683 & n.d. & 0.726 & n.d. & n.d. & 0.345 & n.d. \\
\hline THS71515 & China, Xinjiang & $\operatorname{tg} 3$ & G. glabla & 0.991 & 0.223 & 0.598 & 0.030 & 0.160 & 0.022 & n.d. & n.d. & n.d. \\
\hline THS72275 & China, Xinjiang & $\operatorname{tg} 3$ & G. glabla & 2.275 & 0.049 & 0.937 & n.d. & 0.383 & 0.070 & n.d. & 0.159 & n.d. \\
\hline THS72276 & China, Xinjiang & $\operatorname{tg} 3$ & G. glabla & 3.080 & 0.352 & 1.784 & 0.077 & 0.519 & n.d. & n.d. & 0.157 & n.d. \\
\hline THS72277 & China, Xinjiang & $\operatorname{tg} 3$ & G. glabla & 2.984 & 0.308 & 1.859 & 0.070 & 0.629 & 0.023 & n.d. & 0.099 & n.d. \\
\hline THS72278 & China, Xinjiang & $\operatorname{tg} 3$ & G. glabla & 1.766 & 0.148 & 0.845 & n.d. & 0.293 & n.d. & n.d. & 0.207 & n.d. \\
\hline THS72279 & China, Xinjiang & $\operatorname{tg} 3$ & G. glabla & 4.867 & 0.660 & 2.759 & 0.165 & 0.813 & 0.045 & n.d. & 0.259 & n.d. \\
\hline THS40620 & China, Xinjiang & $\operatorname{tg} 5$ & G. inflata & 2.401 & 0.363 & 0.806 & 0.068 & 0.280 & 0.038 & n.d. & n.d. & 0.686 \\
\hline THS40630 & China, Xinjiang & $\operatorname{tg} 5$ & G. inflata & 2.540 & 0.084 & 0.841 & 0.011 & 0.321 & n.d. & n.d. & n.d. & 1.820 \\
\hline THS60203 & China, Xinjiang & $\operatorname{tg} 5$ & G. inflata & 1.129 & 0.264 & 0.843 & 0.038 & 0.208 & 0.026 & n.d. & n.d. & n.d. \\
\hline THS72281 & China, Xinjiang & $\operatorname{tg} 5$ & G. inflata & 5.380 & 0.385 & 2.291 & 0.059 & 0.855 & n.d. & n.d. & n.d. & 0.634 \\
\hline THS72282 & China, Xinjiang & $\operatorname{tg} 5$ & G. inflata & 3.771 & 0.195 & 1.663 & 0.026 & 0.600 & 0.078 & n.d. & n.d. & 0.762 \\
\hline THS72283 & China, Xinjiang & $\operatorname{tg} 5$ & G. inflata & 5.320 & 0.465 & 2.445 & 0.065 & 0.866 & n.d. & n.d. & n.d. & 0.659 \\
\hline THS72284 & China, Xinjiang & $\operatorname{tg} 5$ & G. inflata & 4.143 & 0.296 & 1.947 & 0.037 & 0.648 & n.d. & n.d. & n.d. & 1.037 \\
\hline THS71518 & China, Gansu & $\operatorname{tg} 5$ & G. inflata & 1.453 & 0.119 & 0.959 & 0.020 & 0.297 & 0.021 & n.d. & n.d. & 0.338 \\
\hline THS58482 & Russia & $\operatorname{tg} 7$ & G. uralensis & 6.178 & 2.984 & 2.975 & 0.799 & 1.219 & 0.084 & n.d. & n.d. & n.d. \\
\hline THS40627 & China, Xinjiang & $\operatorname{tg} 7$ & G. uralensis & 0.850 & 0.282 & 0.557 & 0.050 & 0.157 & 0.008 & n.d. & n.d. & n.d. \\
\hline THS64165 & China, Gansu & $\operatorname{tg} 7$ & G. uralensis & 6.721 & 3.110 & 1.069 & 0.675 & 0.347 & 0.134 & 0.139 & n.d. & n.d. \\
\hline THS72299 & China, Gansu & $\operatorname{tg} 7$ & G. uralensis & 2.744 & 1.876 & 1.016 & 0.330 & 0.315 & 0.115 & 0.139 & n.d. & n.d. \\
\hline THS58610 & China, Ningxia & $\operatorname{tg} 7$ & G. uralensis & 1.847 & 0.449 & 1.859 & 0.069 & 0.604 & 0.061 & 0.070 & n.d. & n.d. \\
\hline THS72303 & China, Ningxia & $\operatorname{tg} 7$ & G. uralensis & 3.008 & 2.153 & 0.390 & 0.444 & 0.094 & 0.108 & 0.031 & n.d. & n.d. \\
\hline THS72305 & China, Ningxia & $\operatorname{tg} 7$ & G. uralensis & 2.658 & 0.659 & 1.418 & 0.136 & 0.607 & 0.029 & 0.078 & n.d. & n.d. \\
\hline THS58144 & China, Shaanxi & $\operatorname{tg} 7$ & G. uralensis & 4.519 & 1.371 & 1.646 & 0.261 & 0.704 & 0.068 & 0.033 & n.d. & n.d. \\
\hline THS58600 & China, Shaanxi & $\operatorname{tg} 7$ & G. uralensis & 2.475 & 1.773 & 0.699 & 0.329 & 0.160 & 0.138 & 0.055 & n.d. & n.d. \\
\hline THS59991 & China, Shaanxi & $\operatorname{tg} 7$ & G. uralensis & 1.966 & 1.303 & 1.118 & 0.184 & 0.277 & 0.063 & 0.101 & n.d. & n.d. \\
\hline THS72285 & $\begin{array}{l}\text { China, } \\
\text { Nei Monggol }\end{array}$ & $\operatorname{tg} 7$ & G. uralensis & 3.364 & 1.612 & 0.694 & 0.299 & 0.179 & 0.164 & n.d. & n.d. & n.d. \\
\hline THS72286 & $\begin{array}{l}\text { China, } \\
\text { Nei Monggol }\end{array}$ & $\operatorname{tg} 7$ & G. uralensis & 6.183 & 4.061 & 2.407 & 0.753 & 0.747 & 0.279 & 0.188 & n.d. & n.d. \\
\hline THS42161 & China, Hebei & $\operatorname{tg} 7$ & G. uralensis & 2.230 & 1.432 & 1.399 & 0.233 & 0.407 & 0.050 & 0.143 & n.d. & n.d. \\
\hline THS72290 & China, Liaoning & $\operatorname{tg} 7$ & G. uralensis & 2.010 & 1.593 & 1.331 & 0.302 & 0.416 & 0.072 & 0.025 & n.d. & n.d. \\
\hline THS40621 & China, Xinjiang & $\operatorname{tg} 8$ & G. uralensis & 3.030 & 1.002 & 0.994 & 0.252 & 0.248 & 0.130 & 0.049 & n.d. & n.d. \\
\hline THS40629 & China, Xinjiang & $\operatorname{tg} 8$ & G. uralensis & 2.222 & 1.146 & 1.162 & 0.216 & 0.286 & 0.054 & 0.055 & n.d. & n.d. \\
\hline THS56597 & China, Xinjiang & $\operatorname{tg} 8$ & G. uralensis & 1.996 & 0.076 & 0.857 & 0.013 & 0.281 & 0.070 & n.d. & n.d. & n.d. \\
\hline THS72270 & China, Xinjiang & $\operatorname{tg} 8$ & G. uralensis & 2.628 & 0.477 & 0.713 & 0.151 & 0.213 & 0.026 & 0.045 & n.d. & n.d. \\
\hline THS72271 & China, Xinjiang & $\operatorname{tg} 8$ & G. uralensis & 2.762 & 0.843 & 0.622 & 0.223 & 0.177 & 0.066 & 0.016 & n.d. & n.d. \\
\hline THS72273 & China, Xinjiang & $\operatorname{tg} 8$ & G. uralensis & 2.057 & 0.873 & 0.805 & 0.154 & 0.215 & 0.087 & 0.015 & n.d. & n.d. \\
\hline THS72274 & China, Xinjiang & $\operatorname{tg} 8$ & G. uralensis & 3.228 & 1.327 & 0.894 & 0.228 & 0.197 & 0.065 & 0.018 & n.d. & n.d. \\
\hline THS61198 & China, Gansu & $\operatorname{tg} 8$ & G. uralensis & 2.692 & 0.989 & 1.605 & 0.182 & 0.435 & 0.085 & 0.086 & n.d. & n.d. \\
\hline THS71543 & China, Gansu & $\operatorname{tg} 8$ & G. uralensis & 1.058 & 0.285 & 0.742 & 0.039 & 0.209 & 0.013 & 0.038 & n.d. & n.d. \\
\hline THS72297 & China, Gansu & $\operatorname{tg} 8$ & G. uralensis & 3.165 & 2.070 & 0.738 & 0.492 & 0.200 & 0.285 & 0.130 & n.d. & n.d. \\
\hline THS72302 & China, Ningxia & $\operatorname{tg} 8$ & G. uralensis & 4.085 & 1.512 & 1.424 & 0.307 & 0.585 & 0.047 & 0.074 & n.d. & n.d. \\
\hline THS42506 & $\begin{array}{l}\text { China, } \\
\text { Nei Monggol }\end{array}$ & $\operatorname{tg} 8$ & G. uralensis & 0.595 & 0.139 & 0.425 & 0.023 & 0.115 & 0.011 & 0.054 & n.d. & n.d. \\
\hline THS42508 & $\begin{array}{l}\text { China, } \\
\text { Nei Monggol }\end{array}$ & $\operatorname{tg} 8$ & G. uralensis & 1.130 & 0.635 & 0.384 & 0.136 & 0.120 & 0.099 & 0.073 & n.d. & n.d. \\
\hline THS58985 & $\begin{array}{l}\text { China, } \\
\text { Nei Monggol }\end{array}$ & $\operatorname{tg} 8$ & G. uralensis & 3.854 & 2.727 & 0.856 & 0.357 & 0.251 & 0.066 & 0.171 & n.d. & n.d. \\
\hline THS59002 & $\begin{array}{l}\text { China, } \\
\text { Nei Monggol }\end{array}$ & $\operatorname{tg} 8$ & G. uralensis & 4.514 & 2.060 & 4.216 & 0.329 & 1.260 & 0.120 & 0.064 & n.d. & n.d. \\
\hline THS72295 & $\begin{array}{l}\text { China, } \\
\text { Nei Monggol }\end{array}$ & $\operatorname{tg} 8$ & G. uralensis & 1.126 & 0.159 & 0.861 & 0.032 & 0.223 & 0.042 & 0.054 & n.d. & n.d. \\
\hline THS31537 & China, Hebei & $\operatorname{tg} 8$ & G. uralensis & 5.578 & 4.022 & 1.557 & 0.750 & 0.401 & 0.049 & 0.121 & n.d. & n.d. \\
\hline
\end{tabular}


Table 1. (Continued)

\begin{tabular}{|c|c|c|c|c|c|c|c|c|c|c|c|c|}
\hline \multirow{2}{*}{ Voucher } & \multirow{2}{*}{ Locality } & \multirow{2}{*}{$\begin{array}{l}\text { Geno- } \\
\text { type }\end{array}$} & \multirow{2}{*}{$\begin{array}{c}\text { Species } \\
\text { identified } \\
\text { by } \\
\text { genotype }\end{array}$} & \multicolumn{6}{|c|}{$\begin{array}{l}\text { Contents of the six main } \\
\text { constituents of licorice }(\%)\end{array}$} & \multicolumn{3}{|c|}{$\begin{array}{l}\text { Contents of the species- } \\
\text { specific constituens (\%) }\end{array}$} \\
\hline & & & & $\begin{array}{l}\text { Glycyr- } \\
\text { rhizin }\end{array}$ & Liquiritin & $\begin{array}{l}\text { Liquiritin } \\
\text { apioside }\end{array}$ & $\begin{array}{l}\text { Isoliqui- } \\
\text { ritin }\end{array}$ & $\begin{array}{l}\text { Isoliquiritin } \\
\text { apioside }\end{array}$ & $\begin{array}{l}\text { Liquiriti- } \\
\text { genin }\end{array}$ & $\begin{array}{l}\text { Glycycou- } \\
\text { marin }\end{array}$ & Glabridin & $\begin{array}{l}\text { Licochal- } \\
\text { cone A }\end{array}$ \\
\hline THS61625 & China, Jilin & $\operatorname{tg} 8$ & G. uralensis & 4.650 & 2.419 & 1.141 & 0.460 & 0.335 & 0.390 & 0.160 & n.d. & n.d. \\
\hline THS41989 & China, Liaoning & $\operatorname{tg} 8$ & G. uralensis & 1.924 & 1.333 & 0.585 & 0.312 & 0.158 & 0.074 & 0.031 & n.d. & n.d. \\
\hline THS72304 & China, Qinghai & $\operatorname{tg} 9$ & G. uralensis & 3.950 & 3.157 & 1.431 & 0.474 & 0.362 & 0.014 & 0.103 & n.d. & n.d. \\
\hline THS58707 & China, Gansu & $\operatorname{tg} 9$ & G. uralensis & 2.110 & 1.827 & 1.199 & 0.224 & 0.280 & 0.096 & 0.021 & n.d. & n.d. \\
\hline THS61197 & China, Gansu & $\operatorname{tg} 9$ & G. uralensis & 4.739 & 3.269 & 1.506 & 0.638 & 0.387 & 0.081 & 0.235 & n.d. & n.d. \\
\hline THS64188 & China, Gansu & $\operatorname{tg} 9$ & G. uralensis & 4.974 & 1.489 & 1.112 & 0.371 & 0.405 & 0.175 & 0.205 & n.d. & n.d. \\
\hline THS71524 & China, Gansu & $\operatorname{tg} 9$ & G. uralensis & 2.566 & 1.434 & 0.611 & 0.242 & 0.159 & 0.012 & 0.079 & n.d. & n.d. \\
\hline THS71528 & China, Gansu & $\operatorname{tg} 9$ & G. uralensis & 2.319 & 1.229 & 0.400 & 0.160 & 0.101 & 0.007 & 0.101 & n.d. & n.d. \\
\hline THS71531 & China, Gansu & $\operatorname{tg} 9$ & G. uralensis & 2.434 & 1.046 & 0.348 & 0.208 & 0.117 & 0.019 & 0.062 & n.d. & n.d. \\
\hline THS71534 & China, Gansu & $\operatorname{tg} 9$ & G. uralensis & 2.741 & 1.425 & 0.722 & 0.221 & 0.232 & 0.028 & 0.066 & n.d. & n.d. \\
\hline THS71537 & China, Gansu & $\operatorname{tg} 9$ & G. uralensis & 3.083 & 0.912 & 0.821 & 0.139 & 0.301 & 0.064 & 0.083 & n.d. & n.d. \\
\hline THS71540 & China, Gansu & $\operatorname{tg} 9$ & G. uralensis & 2.686 & 1.051 & 0.674 & 0.196 & 0.218 & 0.053 & 0.052 & n.d. & n.d. \\
\hline THS72300 & China, Gansu & $\operatorname{tg} 9$ & G. uralensis & 3.405 & 1.208 & 1.471 & 0.203 & 0.411 & 0.024 & 0.073 & n.d. & n.d. \\
\hline THS72306 & China, Ningxia & $\operatorname{tg} 9$ & G. uralensis & 4.421 & 2.666 & 2.677 & 0.395 & 0.782 & 0.064 & 0.092 & n.d. & n.d. \\
\hline THS58143 & China, Shaanxi & $\operatorname{tg} 9$ & G. uralensis & 2.090 & 0.302 & 1.133 & 0.081 & 0.407 & 0.073 & 0.039 & n.d. & n.d. \\
\hline THS58171 & China, Shaanxi & $\operatorname{tg} 9$ & G. uralensis & 3.435 & 0.810 & 2.156 & 0.173 & 0.800 & 0.046 & 0.100 & n.d. & n.d. \\
\hline THS59992 & China, Shaanxi & $\operatorname{tg} 9$ & G. uralensis & 1.961 & 0.476 & 0.909 & 0.086 & 0.315 & 0.020 & 0.103 & n.d. & n.d. \\
\hline THS66460 & China, Shanxi & $\operatorname{tg} 9$ & G. uralensis & 2.810 & 1.996 & 1.586 & 0.342 & 0.483 & 0.051 & 0.079 & n.d. & n.d. \\
\hline THS42504 & $\begin{array}{l}\text { China, } \\
\text { Nei Monggol }\end{array}$ & $\operatorname{tg} 9$ & G. uralensis & 1.187 & 0.561 & 0.394 & 0.104 & 0.119 & 0.016 & 0.053 & n.d. & n.d. \\
\hline THS58601 & $\begin{array}{l}\text { China, } \\
\text { Nei Monggol }\end{array}$ & $\operatorname{tg} 9$ & G. uralensis & 7.671 & 3.900 & 1.910 & 0.834 & 0.531 & 0.136 & 0.057 & n.d. & n.d. \\
\hline THS58605 & $\begin{array}{l}\text { China, } \\
\text { Nei Monggol }\end{array}$ & $\operatorname{tg} 9$ & G. uralensis & 0.964 & 0.264 & 0.443 & 0.043 & 0.135 & 0.022 & 0.031 & n.d. & n.d. \\
\hline THS58743 & $\begin{array}{l}\text { China, } \\
\text { Nei Monggol }\end{array}$ & $\operatorname{tg} 9$ & G. uralensis & 4.147 & 1.441 & 1.546 & 0.268 & 0.518 & 0.480 & 0.087 & n.d. & n.d. \\
\hline THS58745 & $\begin{array}{l}\text { China, } \\
\text { Nei Monggol }\end{array}$ & $\operatorname{tg} 9$ & G. uralensis & 3.700 & 1.801 & 2.295 & 0.241 & 0.728 & 0.030 & 0.098 & n.d. & n.d. \\
\hline THS58747 & $\begin{array}{l}\text { China, } \\
\text { Nei Monggol }\end{array}$ & $\operatorname{tg} 9$ & G. uralensis & 5.524 & 2.123 & 1.637 & 0.406 & 0.627 & 0.083 & 0.193 & n.d. & n.d. \\
\hline THS58748 & $\begin{array}{l}\text { China, } \\
\text { Nei Monggol }\end{array}$ & $\operatorname{tg} 9$ & G. uralensis & 3.303 & 0.875 & 1.392 & 0.127 & 0.504 & 0.058 & 0.150 & n.d. & n.d. \\
\hline THS58966 & $\begin{array}{l}\text { China, } \\
\text { Nei Monggol }\end{array}$ & $\operatorname{tg} 9$ & G. uralensis & 5.244 & 3.236 & 1.824 & 0.568 & 0.619 & 0.057 & 0.094 & n.d. & n.d. \\
\hline THS58967 & $\begin{array}{l}\text { China, } \\
\text { Nei Monggol }\end{array}$ & $\operatorname{tg} 9$ & G. uralensis & 2.742 & 1.863 & 0.675 & 0.281 & 0.214 & 0.052 & 0.055 & n.d. & n.d. \\
\hline THS58968 & $\begin{array}{l}\text { China, } \\
\text { Nei Monggol }\end{array}$ & $\operatorname{tg} 9$ & G. uralensis & 4.915 & 3.719 & 0.895 & 0.666 & 0.240 & 0.050 & 0.083 & n.d. & n.d. \\
\hline THS58986 & $\begin{array}{l}\text { China, } \\
\text { Nei Monggol }\end{array}$ & $\operatorname{tg} 9$ & G. uralensis & 3.281 & 2.532 & 1.414 & 0.446 & 0.401 & 0.052 & 0.088 & n.d. & n.d. \\
\hline THS58987 & $\begin{array}{l}\text { China, } \\
\text { Nei Monggol }\end{array}$ & $\operatorname{tg} 9$ & G. uralensis & 5.510 & 2.934 & 2.669 & 0.475 & 0.916 & 0.092 & 0.118 & n.d. & n.d. \\
\hline THS60967 & $\begin{array}{l}\text { China, } \\
\text { Nei Monggol }\end{array}$ & $\operatorname{tg} 9$ & G. uralensis & 4.033 & 1.809 & 2.361 & 0.340 & 0.701 & 0.101 & 0.228 & n.d. & n.d. \\
\hline THS64185 & $\begin{array}{l}\text { China, } \\
\text { Nei Monggol }\end{array}$ & $\operatorname{tg} 9$ & G. uralensis & 3.533 & 1.832 & 0.748 & 0.342 & 0.250 & 0.067 & 0.145 & n.d. & n.d. \\
\hline THS65279 & $\begin{array}{l}\text { China, } \\
\text { Nei Monggol }\end{array}$ & $\operatorname{tg} 9$ & G. uralensis & 3.260 & 2.086 & 1.630 & 0.341 & 0.506 & 0.196 & 0.111 & n.d. & n.d. \\
\hline THS65898 & $\begin{array}{l}\text { China, } \\
\text { Nei Monggol }\end{array}$ & $\operatorname{tg} 9$ & G. uralensis & 5.474 & 4.356 & 1.495 & 1.256 & 0.406 & 0.407 & 0.080 & n.d. & n.d. \\
\hline THS72287 & $\begin{array}{l}\text { China, } \\
\text { Nei Monggol }\end{array}$ & $\operatorname{tg} 9$ & G. uralensis & 4.523 & 3.281 & 1.477 & 0.401 & 0.438 & 0.103 & 0.215 & n.d. & n.d. \\
\hline THS72288 & $\begin{array}{l}\text { China, } \\
\text { Nei Monggol }\end{array}$ & $\operatorname{tg} 9$ & G. uralensis & 0.259 & 0.136 & 0.502 & 0.015 & 0.099 & 0.011 & 0.085 & n.d. & n.d. \\
\hline THS72291 & $\begin{array}{l}\text { China, } \\
\text { Nei Monggol }\end{array}$ & $\operatorname{tg} 9$ & G. uralensis & 3.181 & 3.458 & 2.431 & 0.404 & 0.617 & 0.057 & 0.137 & n.d. & n.d. \\
\hline THS72292 & $\begin{array}{l}\text { China, } \\
\text { Nei Monggol }\end{array}$ & $\operatorname{tg} 9$ & G. uralensis & 5.858 & 1.048 & 1.287 & 0.248 & 0.673 & 0.156 & 0.053 & n.d. & n.d. \\
\hline THS72293 & $\begin{array}{l}\text { China, } \\
\text { Nei Monggol }\end{array}$ & $\operatorname{tg} 9$ & G. uralensis & 4.228 & 1.696 & 0.868 & 0.284 & 0.272 & 0.031 & 0.078 & n.d. & n.d. \\
\hline THS72294 & $\begin{array}{l}\text { China, } \\
\text { Nei Monggol }\end{array}$ & $\operatorname{tg} 9$ & G. uralensis & 4.439 & 3.132 & 2.441 & 0.524 & 0.673 & 0.115 & 0.158 & n.d. & n.d. \\
\hline THS72296 & $\begin{array}{l}\text { China, } \\
\text { Nei Monggol }\end{array}$ & $\operatorname{tg} 9$ & G. uralensis & 3.395 & 1.204 & 1.225 & 0.264 & 0.505 & 0.129 & 0.120 & n.d. & n.d. \\
\hline
\end{tabular}


Table 1. (Continued)

\begin{tabular}{|c|c|c|c|c|c|c|c|c|c|c|c|c|}
\hline \multirow{2}{*}{ Voucher } & \multirow{2}{*}{ Locality } & \multirow{2}{*}{$\begin{array}{l}\text { Geno- } \\
\text { type }\end{array}$} & \multirow{2}{*}{$\begin{array}{c}\text { Species } \\
\text { identified } \\
\text { by } \\
\text { genotype }\end{array}$} & \multicolumn{6}{|c|}{$\begin{array}{l}\text { Contents of the six main } \\
\text { constituents of licorice }(\%)\end{array}$} & \multicolumn{3}{|c|}{$\begin{array}{l}\text { Contents of the species- } \\
\text { specific constituens (\%) }\end{array}$} \\
\hline & & & & $\begin{array}{l}\text { Glycyr- } \\
\text { rhizin }\end{array}$ & Liquiritin & $\begin{array}{l}\text { Liquiritin } \\
\text { apioside }\end{array}$ & $\begin{array}{l}\text { Isoliqui- } \\
\text { ritin }\end{array}$ & $\begin{array}{l}\text { Isoliquiritin } \\
\text { apioside }\end{array}$ & $\begin{array}{l}\text { Liquiriti- } \\
\text { genin }\end{array}$ & $\begin{array}{l}\text { Glycycou- } \\
\text { marin }\end{array}$ & Glabridin & $\begin{array}{l}\text { Licochal- } \\
\text { cone A }\end{array}$ \\
\hline THS72307 & $\begin{array}{l}\text { China, } \\
\text { Nei Monggol }\end{array}$ & $\operatorname{tg} 9$ & G. uralensis & 7.009 & 3.234 & 1.827 & 0.764 & 0.637 & 0.637 & 0.044 & n.d. & n.d. \\
\hline THS64732 & China, Hebei & $\operatorname{tg} 9$ & G. uralensis & 8.310 & 3.935 & 3.571 & 0.836 & 1.470 & 0.284 & 0.165 & n.d. & n.d. \\
\hline THS64733 & China, Hebei & $\operatorname{tg} 9$ & G. uralensis & 3.878 & 1.262 & 1.140 & 0.265 & 0.561 & 0.155 & 0.045 & n.d. & n.d. \\
\hline THS64864 & China, Hebei & $\operatorname{tg} 9$ & G. uralensis & 2.962 & 2.457 & 0.783 & 0.467 & 0.202 & 0.210 & 0.077 & n.d. & n.d. \\
\hline THS65441 & China, Hebei & $\operatorname{tg} 9$ & G. uralensis & 3.429 & 1.388 & 1.110 & 0.259 & 0.358 & 0.148 & 0.157 & n.d. & n.d. \\
\hline THS72301 & China, Hebei & $\operatorname{tg} 9$ & G. uralensis & 3.166 & 1.625 & 1.083 & 0.233 & 0.253 & 0.054 & 0.079 & n.d. & n.d. \\
\hline THS72308 & China, Hebei & $\operatorname{tg} 9$ & G. uralensis & 1.927 & 1.811 & 0.560 & 0.277 & 0.125 & 0.041 & 0.057 & n.d. & n.d. \\
\hline THS41990 & China, Liaoning & $\operatorname{tg} 9$ & G. uralensis & 3.267 & 1.846 & 0.830 & 0.378 & 0.242 & 0.085 & 0.092 & n.d. & n.d. \\
\hline THS58141 & China, Liaoning & $\operatorname{tg} 9$ & G. uralensis & 3.381 & 1.011 & 1.356 & 0.211 & 0.451 & 0.161 & 0.137 & n.d. & n.d. \\
\hline THS72298 & $\begin{array}{l}\text { China, } \\
\text { Heilongjiang }\end{array}$ & $\operatorname{tg} 9$ & G. uralensis & 3.304 & 1.842 & 2.007 & 0.289 & 0.552 & 0.101 & 0.209 & n.d. & n.d. \\
\hline THS62089 & China, Jilin & $\operatorname{tg} 9$ & G. uralensis & 2.749 & 0.529 & 1.863 & 0.108 & 0.675 & 0.101 & 0.131 & n.d. & n.d. \\
\hline THS66783 & China, Jilin & $\operatorname{tg} 9$ & G. uralensis & 2.945 & 1.486 & 1.077 & 0.307 & 0.249 & 0.152 & 0.066 & n.d. & n.d. \\
\hline THS72309 & China, Jilin & $\operatorname{tg} 9$ & G. uralensis & 3.482 & 2.183 & 2.434 & 0.253 & 0.731 & 0.063 & 0.061 & n.d. & n.d. \\
\hline THS58598 & China, Liaoning & $\operatorname{tg} 9$ & G. uralensis & 3.396 & 1.932 & 0.578 & 0.451 & 0.153 & 0.469 & 0.080 & n.d. & n.d. \\
\hline THS65901 & China, Liaoning & $\operatorname{tg} 9$ & G. uralensis & 6.165 & 2.892 & 1.218 & 0.892 & 0.392 & 0.609 & 0.095 & n.d. & n.d. \\
\hline THS40622 & $\begin{array}{l}\text { China, } \\
\text { Xinjiang }\end{array}$ & $\begin{array}{l}\mathrm{ADD} \\
\text { (AT) }\end{array}$ & hybrid & 1.517 & 0.741 & 0.816 & 0.115 & 0.170 & 0.027 & 0.043 & n.d. & n.d. \\
\hline THS40624 & $\begin{array}{l}\text { China, } \\
\text { Xinjiang }\end{array}$ & $\begin{array}{l}\text { ADD } \\
\text { (AT) }\end{array}$ & hybrid & 3.741 & 0.538 & 0.459 & 0.172 & 0.215 & 0.076 & 0.048 & n.d. & n.d. \\
\hline THS40626 & $\begin{array}{l}\text { China, } \\
\text { Xinjiang }\end{array}$ & $\begin{array}{l}\mathrm{ADD} \\
\text { (AT) }\end{array}$ & hybrid & 1.656 & 0.318 & 0.360 & 0.074 & 0.120 & 0.051 & n.d. & n.d. & 0.067 \\
\hline THS40628 & $\begin{array}{l}\text { China, } \\
\text { Xinjiang }\end{array}$ & $\begin{array}{l}\text { ADD } \\
\text { (AT) }\end{array}$ & hybrid & 2.352 & 0.232 & 0.968 & 0.042 & 0.374 & n.d. & n.d. & n.d. & 0.813 \\
\hline THS71521 & $\begin{array}{l}\text { China, } \\
\text { Gansu }\end{array}$ & $\begin{array}{l}\mathrm{ADD} \\
(\mathrm{GA})\end{array}$ & hybrid & 1.977 & 0.550 & 0.920 & 0.042 & 0.234 & 0.025 & n.d. & n.d. & 0.361 \\
\hline
\end{tabular}

Tsumura Herbarium Specimen (THS). "n.d.” means no detected. AT or GA means the genotype of $r b c L$ sequence.
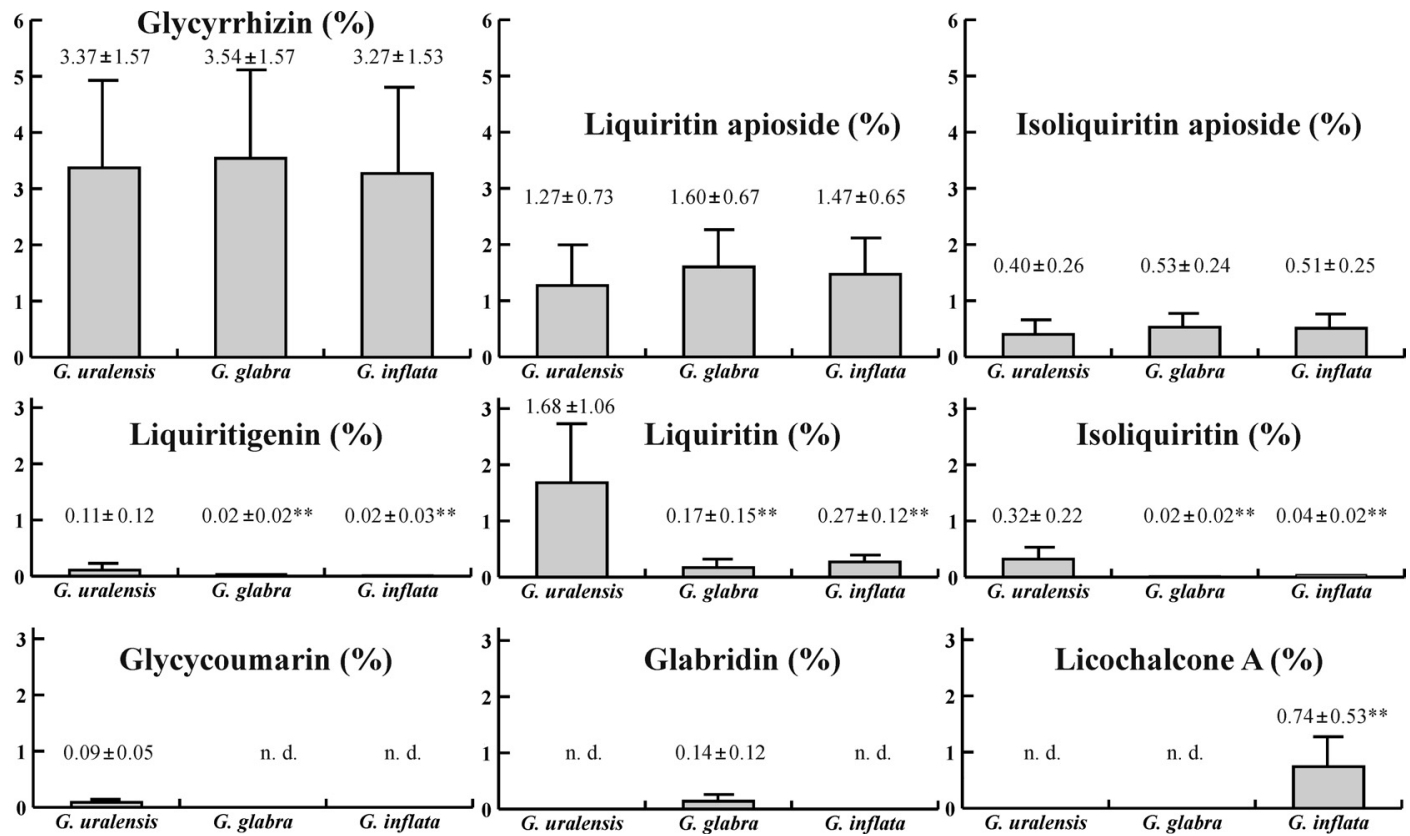

Fig. 1. The Contents of the Six Kinds of Main Constituents by G. uralensis $(n=87)$, G. glabra $(n=17)$, or $G$. inflata $(n=8)$

Values indicate means and S.D. $* * p<0.01$ vs. G. uralensis.

the cytoplasmic gene flow of $r b c L$ gene. Therefore, the determination of the species-specific constituents would be not efficient for the detection of hybrid.

\section{DISCUSSION}

In this study, the constituent properties by the licorices derived from G. uralensis, G. glabra, or G. inflata are revealed. 


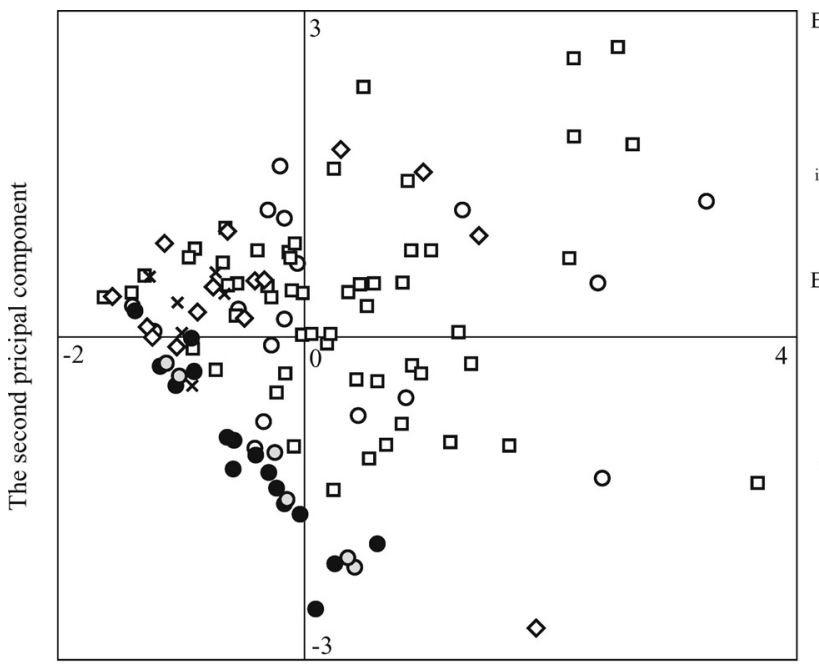

The first pricipal component

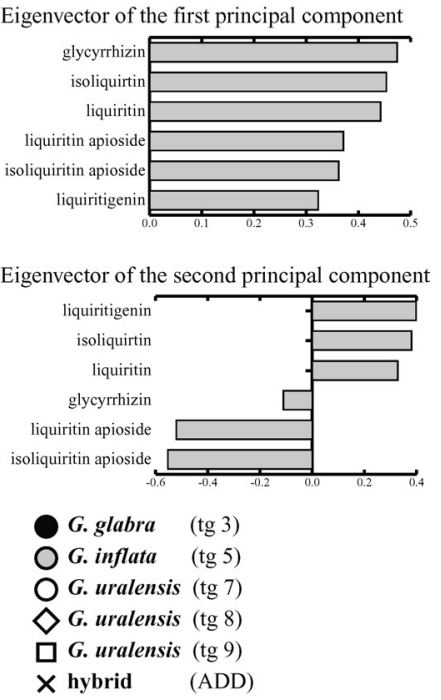

Fig. 2. The Scatter Diagram of the Principal Component Analysis for the Six Kinds of Main Constituent Contents of Licorice Derived from G. uralensis $(n=87)$, G. glabra $(n=17)$, and G. inflata $(n=8)$, and Hybrids $(n=5)$

Those species were identified by their genotypes $(\operatorname{tg} 3, \operatorname{tg} 5, \operatorname{tg} 7, \operatorname{tg} 8, \operatorname{tg} 9$, or ADD).

Table 2. Detection Frequencies of the Three Kinds of Species-Specific Constituents by Species Identified by the Genotypes for 117 Licorices Examined

\begin{tabular}{|c|c|c|c|c|c|}
\hline \multirow{2}{*}{ Genotype } & \multirow{2}{*}{ (species identified) } & \multicolumn{4}{|c|}{ Detecition frequency } \\
\hline & & Glycycoumarin & Glabridin & Licochalcone A & n.d. \\
\hline $\operatorname{tg} 3$ & (G. glabra, $n=17$ ) & 0 & $15(88 \%)$ & 0 & $2(12 \%)$ \\
\hline $\operatorname{tg} 5$ & (G. inflata, $n=8)$ & 0 & 0 & $7(88 \%)$ & $1(12 \%)$ \\
\hline $\operatorname{tg} 7, \operatorname{tg} 8, \operatorname{tg} 9$ & (G. uralensis, $n=87$ ) & $83(95 \%)$ & 0 & 0 & $4(5 \%)$ \\
\hline $\mathrm{ADD}$ & (Hybrid, $n=5$ ) & $2(40 \%)$ & 0 & $3(60 \%)$ & 0 \\
\hline
\end{tabular}

'n.d.' means no detected of the three species-specific constituents.

About the six main constituents of licorice of glycyrrhizin, liquiritin, liquiritin apioside, isoliquiritin, isoliquiritin apioside, and liquiritigenin, the constituent property of $G$. glabra resembles to that of $G$. inflata, but that of $G$. uralensis does not resemble to that of $G$. glabra or G. inflata and is characterized by a wider content variation of the six main constituents of licorice than that of G. glabra or G. inflata. The mean contents of liquiritin, isoliquiritin, and liquilitigenin in G. uralensis are significantly higher than those of $G$. glabra and $G$. inflata. Especially, the mean contet of liquiritin in $G$. uralensis is higher more than $1 \%$ compared to that of $G$. glabra or $G$. inflata. Additionally, glycycoumarin, glabridin, or licochalcone A are reconfirmed as the species-specific constituents for G. uralensis, G. glabra, or G. inflata respectively.

Therefore, when licorice is used for medicinal purposes, the licorice species should be selected with recognition of those constituent properties. In the assessment of the six kinds of main constituent contents, G. glabra and G. inflata can be used equally as medicine, but $G$. uralensis might be not able to use similarly with G. glabra or G. inflata. For example, the mean content of isoliquiritin in $G$. uralensis is significantly higher than that of $G$. glabra or $G$. inflata. Isoliquiritin had inhibitory effects on aldose reductase activity and granuloma angiogenesis. ${ }^{18,19)}$ Hence, licorice species used for Byakko-ka-ninjin-to or Saiko-keishi-kankyo-to in the Japanese Kampo medicinal prescription that are often used as a diabetic medicine ${ }^{20)}$ should be selected under con- sideration of the amount of isoliquiritin in their prescriptions. Additionally, an antitussive effect of liquiritin apioside, liquiritigenin, and liquiritin, and a week estrogen-like activity of liquiritigenin had been reported. ${ }^{21,22)}$

It becomes evident that glabridin, licochalcon A, or glycycoumarin functions as a species-specific constituent of $G$. glabra, G. inflata, or G. uralensis respectively. Therefore, the determination of the three species-specific constituents would be effective for the species identification of licorice. However, since there was no detection of the three speciesspecific constituents from $6 \%$ of licorices examined (7/117) and the hybrids would not be detected from the speciesspecific constituents, the species identification of licorice by the genetic information has high accuracy than by the existence of species-specific constituents.

Acknowledgements We express our sincere thanks to Dr. Cheng Z. and Dr. Pan H. of Xinjiang Research Institute of Ecology and Geography, Chinese Academy of Sciences for providing materials.

\section{REFERENCES}

1) The Pharmacopoeia Commission of People's Republic of China (ed.), "Pharmacopoeia of the Peoples's Republic of China (2005)," Vol. I, Chemical Industry Press, Beijing, 2005, pp. 59-60.

2) The Society of Japanese Pharmacopoeia (ed.), "Japanese Pharmacopoeia," 15th ed., Ministry of Health, Labour and Welfare of Japan, Tokyo, 2001, p. 1197. 
3) Yoneda K., Yamagata E., Tsujimura M., Shoyakugaku Zasshi, 44, 202-206 (1990).

4) Yoneda K., Yamagata E., Teruya M., Shoyakugaku Zasshi, 45, 220226 (1991).

5) Shibano M., Matsumoto Y., Kusano G., Shibata T., J. Nat. Med., 50, $273-283$ (1996)

6) Kitagawa I., Chen W., Taniyama T., Harada E., Hori K., Kobayashi M., Ren J., Yakugaku Zasshi, 118, 519-528 (1998).

7) Shibata S., Saitoh T., J. Indian Chem. Soc., 55, 1184-1191 (1978).

8) Hatano T., Fukuda T., Liu Y., Noro T., Okuda T., Yakugaku Zasshi, 111, 311-321 (1991)

9) Hayashi H., Hosono N., Kondo M., Hiraoka N., Ikeshiro Y., Shibano M., Kusano G., Yamamoto H., Tanaka T., Inoue K., Biol. Pharm. Bull., 23, 602-606 (2000).

10) Ashurmetov O. A., Gen. Res. Crop. Evol., 43, 167-171 (1996).

11) Hayashi H., Hattori S., Inoue K., Sarsenbaev K., Ito M., Honda G., Biol. Pharm. Bull., 26, 867-871 (2003).

12) Hayashi H., Inoue K., Ozaki K., Watanabe H., Biol. Pharm. Bull., 28, 1113-1116 (2005).

13) Kondo K., Shiba M., Yamaji H., Morota T., Takada S., Sasaki H., Cheng Z., Pan H., Shoyama Y., Biol. Pharm. Bull. (in submission).
14) White T. J., Bruns T., Lee S., Taylor J., "PCR protocols, A guide to methods and applications. Amplifications," ed. by Innis M. A., Gelfand D. H., Sninsky J. J., White T. J., Academic Press, San Diego, 1990, pp. 315-322.

15) Demesure B., Sodzi N., Petit J., Molecular Ecology, 4, 129-131 (1995).

16) Kawakami B., Kitabayashi M., Kuroita T., Komatsu S., "PCR tips," ed. by Maki H., Shujunsha, Tokyo, 1999, pp. 189-198.

17) Social Survey Research Information Co., Ltd., "Excel statistics 2002 for windows," Tokyo, 2002.

18) Aida K., Tawata M., Shindo H., Onaya T., Nishimura H., Chin M., Mitsuhashi H., Planta Med., 55, 22-26 (1989).

19) Kobayashi S., Miyamoto T., Kimura I., Kimura M., Biol. Pharm. Bull., 18, 1382-1386 (1995)

20) Hujihira K., Diabetes, "Handbook of Japanese Kampo Medicinal Prescription by Disease and Symptom," ed. by Hujihira Kampo Laboratory, Rinne Press, Tokyo, 1982, pp. 68-69.

21) Kamei J., Satoh A., Asano T., Nakamura R., Ichiki H., Iiduka A., Kubo M., Eur. J. Pharmacol., 10, 163-168 (2005).

22) Nomura T., Fukai T., Akiyama T., Pure Appl. Chem., 74, 1199-1206 (2002). 